\title{
Abnormal post operative bleeding in routine ENT surgery - a caution for the young ENT surgeons
}

\author{
Rojibul Haque ${ }^{1}$, Md. Khalid Asad ${ }^{2}$, MA Rasel ${ }^{3}$, Mani Lal Aich ${ }^{4}$
}

\begin{abstract}
:
Bleeding may complicate many routine ENT procedures. This may result from technical errors, but bleeding associated with coagulopathy may be difficult to control. We report two cases of abnormal post operative bleeding due to coagulopathy, first one admitted on $2^{\text {nd }}$ post operative day after tonsillectomy and second one admitted on $5^{\text {th }}$ post operative day after septoplasty. Case one was diagnosed as a case of Immune Thrombocytopenic Purpura (ITP), and second case was diagnosed later as a case of von Willebrand's Disease. All otolaryngologists should be aware of common bleeding disorders and their treatment. This knowledge will help for appropriate preoperative screening of patients. The most important component of the preoperative assessment is the bleeding history that directs further laboratory evaluation.
\end{abstract}

Key-words: Post operative bleeding, coagulopthy, post tonsillectomy haemorrhage, Immune Thrombocytopenic Purpura (ITP), Von Willebrand's Disease

\section{Introduction:}

Tonsillectomy and septoplasty are the two most frequently performed ENT operations in Bangladesh. These are routine operations without major complications. The main complication is intra-operative and postoperative haemorrhage ${ }^{1}$.

Postsurgical haemorrhage, the major complication of tonsillectomy and a potentially life-threatening occurrence, is classified as primary reactionary $(<24$ hr postoperatively) or secondary ( $>24 \mathrm{hr}$ ). Primary haemorrhage is considered to be more serious than secondary haemorrhage, but that secondary bleeding can also be brisk and massive and require treatment under general anaesthesia ${ }^{2}$.

Bleeding during and after septoplasty, is also distressing both for surgeon and patient. It can be controlled by applying ANS and/or PNS pack, but this causes morbidity, increase time of hospital stay and cost of the procedure.

In both cases, situation may become more complicated in presence of undiagnosed bleeding

1. Assistant Professor, Dept. of ENT/HNS, SSMC\&MH

2. OSD, DGHS, Dhaka

3. Honorary Medical Officer, Dept. of ENT/HNS, BSMMU

4. Assistant Professor, Dept. of ENT/HNS, Dhaka Medical College Hospital

Address of Correspondence to: Dr. Rojibul Haque, House\# 357, Road\#4, Baitul Aman Housing Society, Adabor, Shamaloy, Dhaka -1207, Bangladesh, E-mail: rojibul@yahoo.com disorder. Most bleeding disorders are obvious and are diagnosed prior to surgery. A small number of cases of mild Hemophilia or Von Willebrand's Disease may be uncovered at the time of surgery by observing abnormal bleeding ${ }^{3}$. Patient may also need emergency admission with secondary haemorrhage.

We report two case of post operative bleeding due to bleeding disorder which was previously undiagnosed on preoperative screening.

\section{Case Reports}

\section{Case -1:}

A 12-year-old boy was admitted in the Dept. of ENT of Dhaka Medical College Hospital with the complaints of bleeding form tonsiller fossa for two days. He had tonsillectomy operation, performed 3 days back in a private hospital of a district near Dhaka. As per operation note, the surgeon who performed this operation experienced unusual bleeding during the procedure. It was diffuse, coming from both tonsiller fossa and was difficult to control. Haemostasis was done by suturing the bleeding point and using bipolar diathermy and patient was shifted to recovery room. Within a few hours bleeding started again. There were slight but continuous oozing from both fossa. Measures were taken to control but failed. Two units fresh blood were transfused and patient was referred to $\mathrm{DMCH}$.

On admission detail history was taken from the patient's father and examined thoroughly. He had a 
history of chronic tonsillitis for 5 years. His preoperative hematological workup including CBC, Bleeding time, clotting time was within normal limit and had no history of abnormal bleeding.

On examination, there were clot in both tonsiller fossas. After removing the clot no specific bleeding point was found, but there was continuous oozing from both fossas. Two gauges soaked with adrenaline and hydrogen per oxide was applied to the fossas. Bleeding stopped for some time but started again. Blood transfusion was started. Blood was sent for CBC, Bleeding time (BT), clotting time, Prothombin time (PT), activated partial thromboplastin time (aPTT). Bleeding time and aPTT were raised, platelet count was 1,50,000/ $\mathrm{cmm}$. The patient was then reffered to hematology dept. They again perform total count, BT and aPTT. Platelet count was $20,000 / \mathrm{cmm}$, BT and aPTT were raised. Next day platelet count was $10,000 / \mathrm{cmm}$. They diagnosed him as a case of Idiopathic or Immune Thrombocytopenic Purpura (ITP), and advised for fresh blood transfusion, per-enteral methylprednisolone, and antibiotics. The treatment started immediately, the condition was improved and the bleeding was stopped within next two days. The patient was kept in observation for another five days and then discharged on $7^{\text {th }}$ day of admission. His follow up examination was uneventful, platelet count returned to normal level.

\section{Case -2:}

A 32-year-old man was admitted in the Dept. of ENT of $\mathrm{DMCH}$ with history of septoplasty operation 5 days back in a district hospital. After removing nasal pack on $2^{\text {nd }}$ post operative day, profuse bleeding started from both nose. It was not controlled with ice pack and pressure, ANS pack was reapplied. After 48 hours, pack was removed, but still there were bleeding in nasopharynx. Patient was transfused two units' blood, ANS pack applied and referred to $\mathrm{DMCH}$.

On admission at $\mathrm{DMCH}$, detail history was taken. Patient gave positive history of bleeding disorder, which he didn't mention to his surgeon preoperatively. $\mathrm{He}$ also gave positive family history for bleeding disorder. He experienced excessive bleeding during his circumcision at childhood and also experienced excessive bleeding after an incidence of RTA.

On examination, ANS pack was insitu, slight oozing seen in the nasopharynx during deglutition. He was moderately anaemic, pulse 110/min, BP 100/60 mm $\mathrm{Hg}$. His routine preoperative investigations were all within normal limit.

Blood transfusion was started, blood sent for haematological analysis. Reports showed moderately anaemic, bleeding time was raised, APTT also raised. Hematology consultation was obtained to further define this bleeding disorder. A von Willebrand's profile and factor IX level were obtained. Factor IX level and Factor VIII:C were normal. The Factor VIII:VW was decreased to $37 \%$ (51-151\%) consistent with Type 1 von Willebrand's Disease. Initial treatment consisted of close observation, trauma precautions, and factor VIII infusion for three days. Pack removed after 72 hours, bleeding stopped and the patient discharged with no further bleeding and advised to follow up at hematology deptartment.

\section{Discussion:}

Every patient should be evaluated for bleeding disorder prior to any surgery. Which requires thorough history and physical exam. Suspicion of a bleeding disorder is often initiated by an abnormal laboratory test, but history and physical exam are very effective. Important questions include prolonged bleeding following minor trauma especially after circumcision, spontaneous bruising, or hematoma formation. Any history of excessive bleeding with prior surgical procedures may indicate a bleeding disorder. Drug history is important since many drugs cause bleeding. Aspirin and other cycloxygenase inhibitors are the most common drugs associated with bleeding disorders. ${ }^{4}$ Each patient should be questioned regarding family history and any possible pattern of inheritance. The physical exam may provide important clues as well. Petechiae and purpura are signs of platelet dysfunction.

Normal coagulation may be divided into three phases: vascular response, platelet activation, and the coagulation cascade. Bleeding disorders may be divided into vascular, platelet, coagulation factor, and multiple compartment defects. Vascular disorders may be hereditary or acquired. Laboratory tests are characterized by a prolonged bleeding time. Platelet disorders are characterized by petechiae, purpura, mucosal membrane bleeding, and bruisibility. Acquired platelet disorders include immune thrombocytopenic purpura; drug induced infectious thrombocytopenia; and renal failure. Viral infections are a common cause of immune and infectious thrombocytopenia. Aspirin and other cyclooxygenase inhibitors are the most common cause of drug induced platelet dysfunction. This occurs due to the inhibition of synthesis of thromboxane A potent stimulator of platelet activation. ${ }^{5}$

Hemophilia A and von Willebrand's Disease are the most common congenital coagulation disorders and both involve different components of factor VIII macromolecule. Hemophilia A or classic hemophilia is inherited as a sex-linked recessive and is a 
deficiency or defect in factor VIII:C. Von Willebrand's Disease is characterized by a deficiency or dysfunction of the Factor VIII:VW resulting in impaired platelet adhesion. This disorder is inherited as a autosomal dominant and is characterized by bleeding from mucocutaneous surfaces. Symptoms vary tremendously with each patient, but excessive bleeding may occur with trauma or surgery. Treatment is with cryoprecipitate replacement. ${ }^{6}$

Preoperative screening for bleeding disorders remains a very controversial subject. Most bleeding disorders are obvious and are diagnosed prior to surgery. Asmall number of cases of mild Hemophilia or Von Willebrand's Disease may be uncovered at the time of surgery. ${ }^{7}$ Basic laboratory evaluation of coagulation disorders consists of platelet count, prothrombin time, partial thromboplastin time, and bleeding time. Other more specialized tests include qualification of specific factor levels and tests of platelet function. ${ }^{8}$ Platelet count is an important initial test since thrombocytopenia is the most common cause of bleeding. The bleeding time is an in vivo assessment of platelet response to limited vascular injury and is reflective of the integrity of the primary hemostatic mechanism. Bleeding time is prolonged in thrombocytopenia, qualitative platelet disorders, von Willebrand's Disease, fibrinolytic states, afibrinogenemia, vasculitis, and aspirin therapy. The partial thromboplastin time tests all factors of the classic intrinsic cascade and all factors of the extrinsic cascade with the exceptions of factor VII. The PT tests the extrinsic pathway, in particular vitamin $\mathrm{K}$ dependent factors X, VII, V, II, and fibrinogen. Specific factor levels may be tested as well. The most common factor to be tested is Factor VIII. Factor IX is often commonly measured since Hemophilia $\mathrm{C}$ is the third most common inherited bleeding disorder. ${ }^{9}$

Our first patient was diagnosed as a case of ITP. There are very few reported cases of post tonsillectomy haemorrhage due to ITP. Patient's preoperative investigations showed no abnormality, even gave no positive bleeding history. ITP can present acutely in otherwise healthy child, presented with sudden onset of petechiae, purpura, or epistaxis. It typically starts after a viral disease. Usually it resolves spontaneously, and if symptom less required no treatment. If platelet counts become less than $50000 / \mathrm{cmm}$, steroid therapy is recommended ${ }^{10}$. Probably this patient had recent viral infection, which precipitates the thrombocytopenia.

The second patient was suffering from congenital factor VIII deficiency or von Willebrand's Disease, which was missed by the surgeon during his preoperative evaluation. Patient also didn't disclose his prior bleeding history. His preoperative bleeding time was 8 min, which was within normal limit. Probably his factor VIII activity was within normal range (51\% - $151 \%)$ at the time of investigation.

\section{Conclusion:}

Bleeding during and after a routine ENT surgery is very common and sometime unavoidable. But in case of abnormal and excessive bleeding, pre-existing bleeding disorder should be excluded. Preoperative screening, a careful bleeding history is used to identify any potential bleeding disorders and guide further laboratory test. Failure to recognize an uncommon acquired coagulation disorder can have serious consequences. Our cases illustrate that patients presenting with bleeding disorders can have normal routine coagulation test results. So this is a caution for young ENT surgeons, that they should be very careful about pre operative and haemostatic evaluation.

\section{References:}

1. Capper JW, Randall C. Post-operative haemorrhage in tonsillectomy and adenoidectomy in children. J Laryngol Otol 1984; 989: 363-365.

2. Caromdy D, Vamadevan T, Cooper Sm. Post tonsillectomy haemorrhage. J Laryngol Otol 1982; 96: 635-638.

3. Blombäck $M$, Johansoon $G$, Johnsson $H$, Swedenborg J, Wabö E. Surgery in patients with von Willebrand's disease. Br J Surg 1989;76:398400.

4. Allison PM. Hemostasis. In: Sandstad JS, McKenna RW, Kerref JH, eds. Handbook of Clinical Pathology. Chicago: American Society of Clinical Pathologists, 1992:229-244.

5. Bick TL. Disorders of Hemostasis and Thrombosis: Principles of Clinical Practice. New York: Thieme, 1985.

6-7. Gumprecht TF, Cichon VJ. Otolaryngology and von Willebrand's disease. Arch Otolaryngol 1981;107:491-493.

9-10. Hutchinson RE, Davey FR, Henry JB. Evaluation of coagulation disorders in patients with diseases of Waldeyer's ring. Otolaryngol Clin North Am 1987;20:317-330. 